\title{
Cosmogenic Neutrinos Challenge the Cosmic Ray Proton Dip Model
}

\section{Jonas Heinze*}

Deutsches Elektronen-Synchrotron (DESY), Platanenallee 6, 15738 Zeuthen, Germany

E-mail: jonas.heinze@desy.de

\section{Denise Boncioli}

Deutsches Elektronen-Synchrotron (DESY), Platanenallee 6, 15738 Zeuthen, Germany

E-mail: denise.boncioliedesy.de

\section{Mauricio Bustamante}

Center for Cosmology and AstroParticle Physics (CCAPP), The Ohio State University,

Columbus, OH 43210, USA

Department of Physics, The Ohio State University, Columbus, OH 43210, USA

E-mail: bustamanteramirez.1@osu.edu

\section{Walter Winter}

Deutsches Elektronen-Synchrotron (DESY), Platanenallee 6, 15738 Zeuthen, Germany

E-mail: walter.winter@desy.de

The origin and composition of ultra-high-energy cosmic rays (UHECRs) remain a mystery. The proton dip model describes the shape of the cosmic ray spectrum above $10^{9} \mathrm{GeV}$ by the effect of a pure proton spectrum propagating through the cosmic microwave background. In these interactions secondary neutrinos are produced.

We fit the recent UHECR spectrum measurements from the Telescope Array experiment under the assumption of pure proton composition, as assumed by the proton dip model.

We present a full scan of the three main physical model parameters of UHECR-injection: source redshift evolution, injected maximal proton energy and spectral power-law index. We discuss how the result qualitatively changes compared to earlier two-parameter fits in the literature: a mild preference for a maximal energy cutoff at the sources instead of the Greisen-Zatsepin-Kuzmin (GZK) cutoff, hard injection spectra, and strong source evolution.

We show that the predicted neutrino flux exceeds the IceCube limit for any parameter combination. As a result, the proton dip model is challenged at more than 99\% C.L. This is strong evidence against the dip model independent of mass composition measurements.

35th International Cosmic Ray Conference - ICRC2017

10-20 July, 2017

Bexco, Busan, Korea

\footnotetext{
* Speaker.
} 


\section{Introduction}

Ultra-high-energy cosmic rays (UHECRs) are charged particles of astrophysical origin with energies above $10^{9} \mathrm{GeV}$, the highest observed. Their sources are unknown, but their energy spectrum has been measured with increasing precision $[1,2]$. It exhibits a hardening at about $5 \times 10^{9}$ $\mathrm{GeV}$ - the "ankle" - and a strong suppression at the topmost energies, around $5 \times 10^{10} \mathrm{GeV}-$ the Greisen-Zatsepin-Kuzmin (GZK) cutoff.

If UHECRs above $10^{9} \mathrm{GeV}$ are mainly protons of extragalactic origin, the spectral features can be attributed to interactions with the cosmic microwave background (CMB) and the infrared/optical photon background (CIB). In this "proton dip" model, energy losses due to electron-positron pair production on $\mathrm{CMB}$ photons are responsible for the ankle and photohadronic interactions lead to the GZK-cutoff $[3,4,5]$. Additionally, photohadronic interactions produce secondary cosmogenic neutrinos. If UHECRs are a mixture of nuclei, the interpretation of spectral features is more intricate and cosmogenic neutrinos will be supressed. However, the spectral shape alone cannot distinguish these two scenarios.

The largest UHECR observatories - the Pierre Auger Observatory [6] and the Telescope Array (TA) [7] - also aim to measure the mass composition of CRs. They detect UHECR-initiated extensive air showers via surface Cherenkov water tanks or scintillators, fluorescence detectors, or a combination of both techniques. UHECR mass composition is determined chiefly by measuring the column depth in the atmosphere at which the particle content of a shower is maximal, $\left\langle X_{\max }\right\rangle$.

While TA finds consistency with a light primary composition above $10^{9} \mathrm{GeV}$ [8], Auger finds that the mass of the primary reaches a minimum around $10^{9.3} \mathrm{GeV}$ before rising with energy [9]. However, these conclusions rely on predictions of air shower models: While the $\left\langle X_{\max }\right\rangle$ measurements of the two experiments agree within systematic uncertainties, the different interpretations of composition are dependent on the respective shower models [10].

In this paper, which is based on [11], we test the proton dip model in a complementary approach: By fitting the UHECR spectrum measurements from the TA Collaboration, comprising 7 years of data [2], we obtain a normalization on the flux of cosmogenic neutrinos. This flux should not exceed the recent upper bound from the IceCube Collaboration [12]. We find a compelling conclusion: the high cosmogenic neutrino fluxes implied by TA data challenge the proton dip model at $>95 \%$ C.L., for any parameter combination.

\section{Model and methods}

Our fundamental assumption is that of an extragalactic pure-proton UHECR composition above $10^{9} \mathrm{GeV}$. The main parameters of our cosmic ray transport model are the spectral injection index $\gamma$, maximal proton energy reached via acceleration at the source $E_{\max }$, and source evolution parameter $m$. We assume a homogeneous distribution of identical sources injecting protons (in the cosmologically co-moving frame)

$$
J_{p}^{\mathrm{inj}}(E) \propto H(z) E^{-\gamma} \exp \left(-E / E_{\max }\right),
$$

where $E$ is the energy of the injected protons. The dimensionless function $H(z)$ implements the redshift evolution of the number density of sources, normalized by fixing $H(0)=1$. 
A frequently used assumption is $H(z) \propto(1+z)^{k}$, for small $z$. This is appropriate for UHECR propagation dominated by low redshifts. However, higher redshifts $z>1$ significantly contribute to the flux of cosmogenic neutrinos, and the above parameterization would potentially overproduce neutrinos. We instead parameterize the source evolution relative to the star formation rate (SFR) given in [13], i.e., $H(z)=(1+z)^{m} \times H_{\mathrm{SFR}}(z)$.

We simulate injection up to $z=6$. We allow for positive and negative values of $m: m=0$ corresponds to SFR evolution; $m=-3.4$, to no evolution locally; and $m<-3.4$, to negative evolution [14]. Typically, however, $m>0$ to account for the fact that the luminosity of a source class was higher in the past.

The proton propagation is computed numerically via a transport equation that includes adiabatic, pair production, and photohadronic energy losses; for details, see Appendix B in [15]. We use the CIB at $z=0$ from [16] and scale it by the SFR at higher redshifts. The effects of magnetic fields are not included, which is a good approximation above $10^{9} \mathrm{GeV}$ [5]. We adopt a $\Lambda \mathrm{CDM}$ cosmology with $\Omega_{m}=0.27, \Omega_{\Lambda}=0.73$ and $H_{0}=70.5 \mathrm{~km} \mathrm{~s}^{-1} \mathrm{Mpc}^{-1}$.

We scan over combinations of $\gamma, \log _{10}\left(E_{\max } / \mathrm{GeV}\right)$, and $m$. For each combination, we fit the computed proton flux at Earth to the latest (7-year) combined - surface and fluorescence - energy spectrum measured by the TA Collaboration [2] starting from $10^{9.2} \mathrm{GeV}$. Note that the ankle is fully included in that energy range, and that the transition energy is high enough that magnetic field effects on the cosmic ray propagation can be neglected.

For each parameter combination, the free normalization $f$ and the shift in the energy scale $\delta_{E}$ are found by minimizing the $\chi^{2}$ estimator

$$
\chi^{2}=\sum_{i} \frac{\left(f J_{p}^{\bmod }\left(E_{i}^{\prime} ; \gamma, E_{\max }, m\right)-J_{p}^{\mathrm{TA}}\left(E_{i}\right)\right)^{2}}{\sigma_{i}^{2}}+\left(\frac{\delta_{E}}{\sigma_{E}}\right)^{2},
$$

where $J_{p}^{\bmod }\left(E_{i}^{\prime} ; \gamma, E_{\max }, m\right)$ is the calculated (model), unnormalized flux at Earth, evaluated at the shifted energy $E_{i}^{\prime} \equiv\left(1+\delta_{E}\right) E_{i}$, and $J_{p}^{\mathrm{TA}}\left(E_{i}\right)$ is the measured flux at energy $E_{i}$. At $E_{i}$, the statistical error is $\sigma_{i}$. The sum is performed over the $21 \mathrm{TA}$ data points above $10^{9.2} \mathrm{GeV}$. The last term is a penalty from systematics. The best fit is obtained at minimum $\chi^{2}$ over all model parameters, which indicates the goodness of fit, whereas $\Delta \chi^{2}=\chi^{2}-\chi_{\min }^{2}$ determines the allowed regions. Both $f$ and $\delta_{E}$ are considered nuisance parameters, while the physical parameters are $\gamma, E_{\max }$, and $m$.

\section{3D fits of UHECR parameters}

We first perform 2D scans, fixing one of the physical parameters, to compare our results with the existing literature. It is shown on the left side of Fig.1. In the upper panel, we fix $m=0$, corresponding to SFR evolution. The lower left panel, shows the result of a fit with fixed $\gamma=2.45$. In the lower left panel, we instead fix $E_{\max }=10^{12} \mathrm{GeV}$. The corresponding best-fit parameters are reported in Table 1 . The regions are relatively small and compatible with similar results in the literature $[17,18,19]$. Large $E_{\max }$ and soft injection spectra are preferred. This is by construction, since we chose $E_{\max }$ large in the lower right panel, and the other fixed values were picked from it. We will see this change in the 3D scan. 

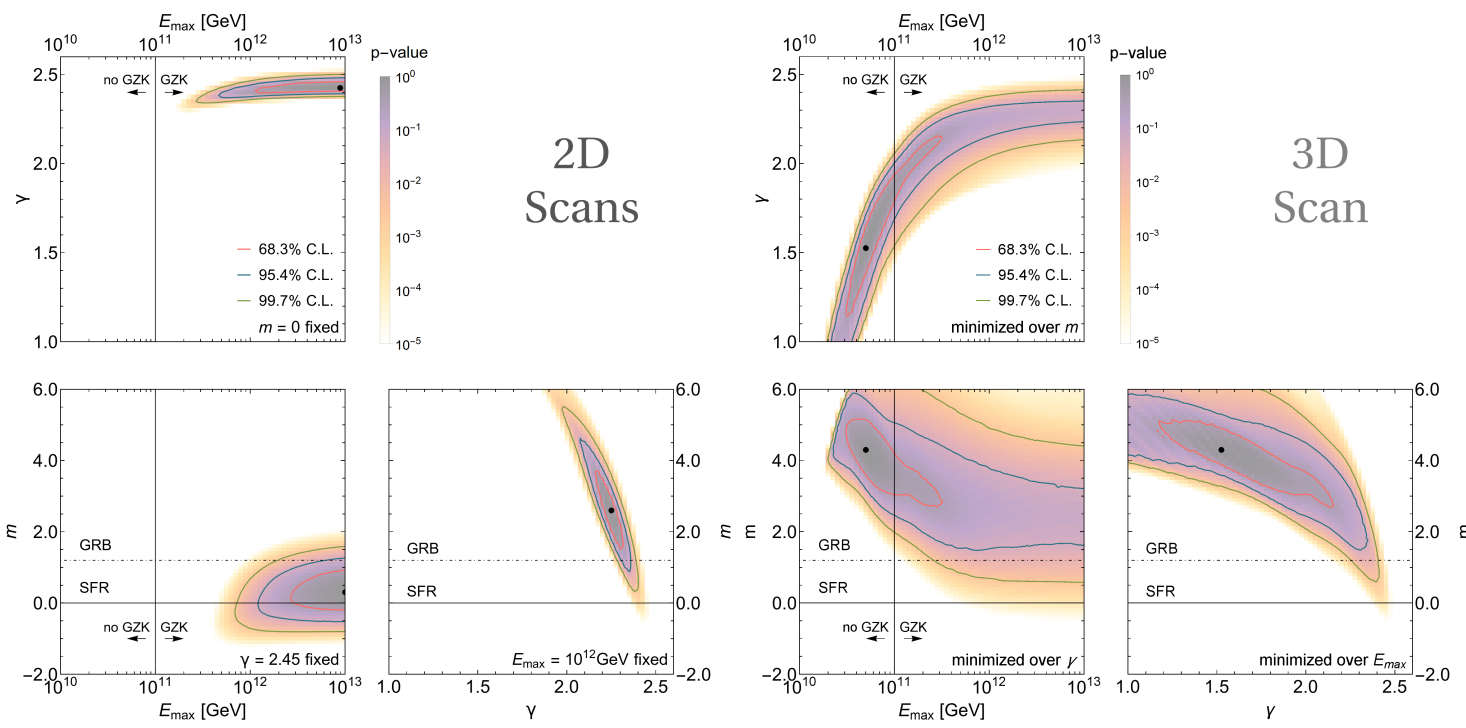

Figure 1: Figures taken from [11]. Allowed two-parameter regions (2 d.o.f.) from 2D fits and 3D fit to the TA spectral data [2]. Left: The third parameter is kept fixed to the value shown in each panel. Right: In each panel, the third parameter has been minimized over.

\begin{tabular}{ccccc}
\hline & \multicolumn{3}{c}{ 2D scans } & 3D scan \\
\hline$\gamma$ & 2.25 & $* 2.45$ & 2.42 & $1.52_{-0.20}^{+0.35}$ \\
$\log _{10}\left(E_{\max } / \mathrm{GeV}\right)$ & $* 12.0$ & 13.0 & 12.9 & $10.7_{-0.1}^{+0.3}$ \\
$m$ & 2.6 & 0.3 & $* 0.0$ & $4.3_{-0.8}^{+0.4}$ \\
$\delta_{E}$ & -0.11 & -0.06 & -0.16 & -0.35 \\
$\chi_{\min }^{2} /$ d.o.f. & $34.7 / 17$ & $47.8 / 17$ & $47.8 / 17$ & $30.8 / 16$
\end{tabular}

Table 1: Best-fit parameters, $1 \sigma$ uncertainties (for the 3D scan only), and $\chi_{\min }^{2} /$ d.o.f. for the $2 \mathrm{D}$ scans and for the $3 \mathrm{D}$ scan. The cases marked with * refer to the fixed parameter in the $2 \mathrm{D}$ scans.

Fig. 1 also shows our results for the 3D scan in $\left(\gamma, E_{\max }, m\right)$, as projections onto three different planes. Compared to the 2D scan, the regions are larger due to multi-parameter correlations. The result is qualitatively different from the 2D scans and from previous literature. The best-fit values of the parameters and their uncertainties are reported in Table 1 . The value of $\chi_{\min }^{2} /$ d.o.f. $=30.8 / 16$ is an improvement over our $2 \mathrm{D}$ scans.

The 3D fit slightly prefers lower maximal energies, harder spectra, and stronger source evolution. The high value of $m$ implies that the contribution of distant sources is enhanced with respect to SFR evolution. The interpretation of the flux suppression at the highest energies as due to the maximal injected energy at the sources is slightly favored over the GZK cutoff interpretation. However, the $\chi^{2}$ function is relatively flat and extended in parameter space, so the preference is mild.

In the 2D scans one parameter was fixed in each panel. By including it in the fit, the allowed regions are extended. They maintain their flat behavior with $E_{\max }$ above $\sim 3 \times 10^{11} \mathrm{GeV}$. The comparison between the 3D scan minimized over $E_{\max }$ and the 2D scan with fixed $E_{\max }=10^{12} \mathrm{GeV}$ 
shows that this choice for the maximal energy restricts the allowed parameter space $(\gamma, m)$ to a small region where the fit can only select a combination of high values for $\gamma$ and mid-to-high values for $m$ (lower right panels). By letting $E_{\max }$ float, many more combinations of $\gamma$ and $m$ become accessible. The spectrum hardens, while the source evolution increases in order to enhance the contribution of distant sources. This happens at the expense of the maximal energy, whose allowed region now extends below the GZK cutoff.

Fig. 2, left panel, shows the best-fit proton spectra at Earth, for the 3D scan (solid) and the 2D scans (dashed/dotted). The energy scale of each spectrum has been shifted by $\delta_{E}$ resulting from its own fit. At the highest energies, the fluxes for the 2D scans look rather similar because the maximal energy in these cases is larger than the GZK threshold. In contrast, the maximal energy found in the 3D scan is at the level of the GZK threshold, which creates the sharper cutoff at the highest energies, by demanding a large shift in the energy scale.

The effect of source evolution can be clearly seen in the overshooting of the data below $10^{9.2} \mathrm{GeV}$. It is largest for the 2D scan with fixed $E_{\max }$ because of the combination of soft spectral index and non-zero evolution. In the 3D scan, the evolution is stronger, but the harder spectral index allows the low-energy flux to be smaller than in the 2D scan.

Fig. 2, right panel, shows the best-fit proton spectrum at Earth and its confidence intervals (at 1 d.o.f.) for the 3D scan. The minimal and maximal proton spectra at each energy are set by the lower and upper edges of the shaded regions at each energy. The largest variations can be seen below the fitting region. The maximal proton flux in this region has to be ascribed to the highest allowed values for $m$ within the $99.7 \%$ C.L., that are connected with the lowest allowed values for $E_{\max }$, responsible for the minimal proton flux at the highest energies. The maximal proton flux at the highest energies instead corresponds to a larger $E_{\max }$, which is related to a smaller $m$ that is responsible for the minimal proton flux at the lowest energies.
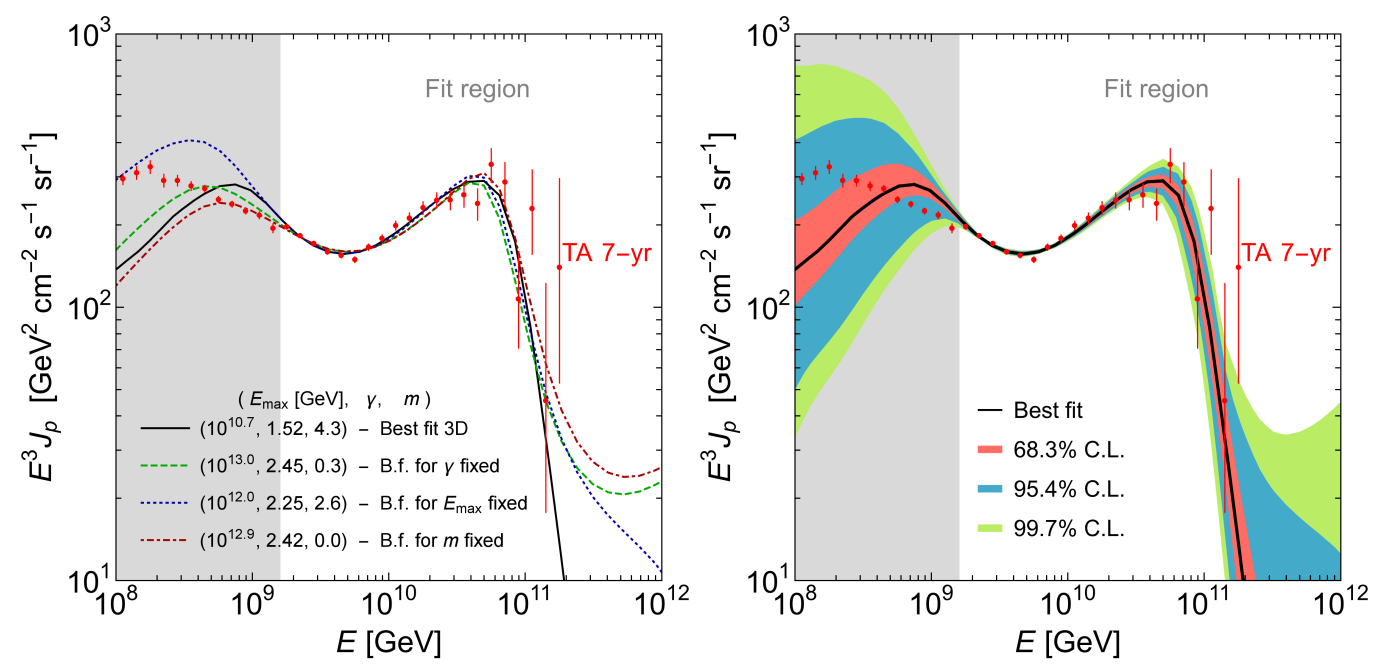

Figure 2: Figures taken from [11]. Left: Best-fit UHECR spectra for 3D scan (solid curve) and 2D scans (dashed/dotted curves), superimposed on the TA 7-year data [2]. The energy scale of the data points is fixed, while that of the models is for each one shifted by the best-fit value of $\delta_{E}$. Right: Bands determined by the minimal and maximal proton spectra corresponding to each confidence level (at 1 d.o.f.) for each energy. 


\section{Cosmogenic neutrinos}

Cosmogenic neutrinos are produced in photohadronic interactions during cosmic-ray propagation. They reach Earth from much higher redshifts than cosmic-rays as they rarely interact and only undergo adiabatic energy losses. If UHECRs are dominated by protons, cosmogenic neutrinos will reach EeV energies; if UHECRs are dominated by nuclei, photomeson production will be supressed and most neutrinos come from neutron decays [20, 21]. Cosmogenic neutrinos remain undetected, but IceCube searches have recently reached the lowest flux sensitivities [12].

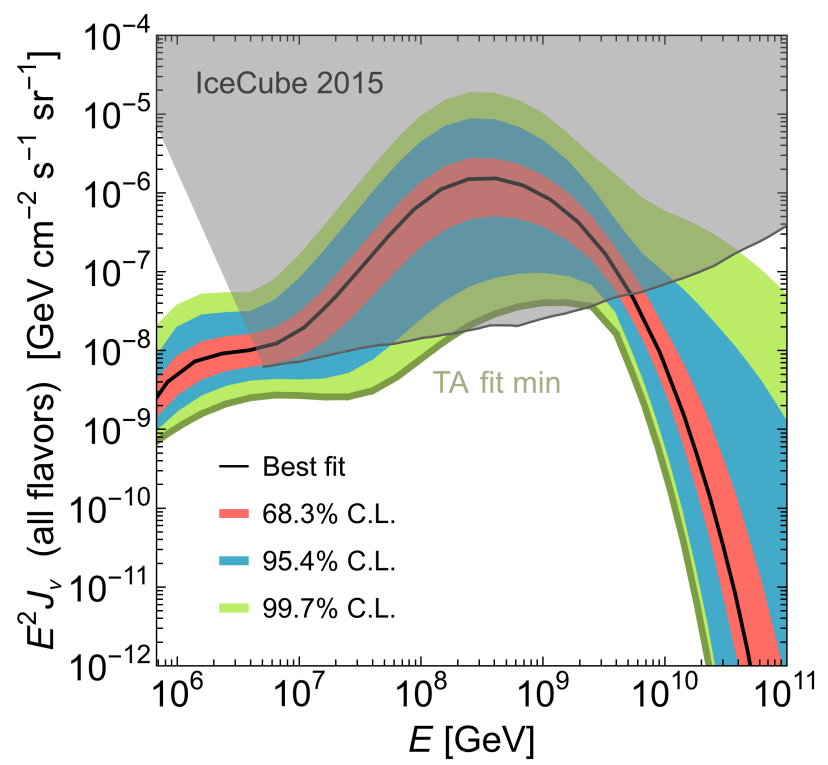

Figure 3: Figure taken from [11]. All-flavor flux of cosmogenic neutrinos predicted by the 3D fit to the TA 7-year UHECR spectrum reported in Section 3. The IceCube upper limit is taken from [12].

By fitting the flux of UHECRs, we also fix the normalization of the associated cosmogenic neutrinos. It should not exceed the IceCube upper limit. Figure 3 shows the flux of cosmogenic neutrinos associated to the best-fit proton spectrum obtained in the 3D scan discussed in Section 3. The shaded bands around it denote confidence intervals (at 1 d.o.f.) corresponding to the cosmic ray fit. Their edges are obtained by finding, at each energy, the minimal and maximal allowed neutrino fluxes within the chosen confidence level, i.e., they are piece-wise dominated by different neutrino spectra.

The bottom curve - marked "TA fit min" - is the envelope of all possible neutrino fluxes allowed by the cosmic ray fit. It is in tension with the TA UHECR data at $99.7 \%$ C.L. Below $\sim 4 \times 10^{9} \mathrm{GeV}$, it corresponds to a larger $E_{\max }$ and a smaller $m$; above, to a smaller $E_{\max }$ and a larger $m$. The proton dip model is disfavored because this minimal envelope exceeds the IceCube upper limit [12].

The number of expected events above $10^{7} \mathrm{GeV}$ associated to the best-fit solution is more than 20 times what has been shown to be the highest expectation for IceCube [21], i.e., it can clearly be ruled out. Most importantly, "TA fit min" yields 5.4 events. Since no event was observed, this flux can be excluded at $95 \%$ C.L. [22]. Given that this is an unphysical, envelope solution, the actual number of expected events from the allowed parameter space is even somewhat higher. 


\section{Summary and conclusions}

We have performed a 3D parameter space scan of the key UHECR parameters: spectral injection index, maximal proton energy, and source redshift evolution, assuming pure proton composition. The 3D fit has qualitatively different features compared to 2D scans previously performed in the literature, due to multi-parameter correlations. An interpretation of the data in terms of hard spectra, strong source evolution, and low maximal proton energy is slightly favored over the conventional GZK cutoff scenario - at the expense of a large systematic shift of the energy scale.

We have also computed the associated cosmogenic neutrino fluxes in the 3D scan. We have identified the minimal allowed neutrino flux ("TA fit min"), corresponding to the $99.7 \%$ C.L. region allowed by the fit to cosmic ray data. It is in tension with the IceCube upper limit at more than $95 \%$ C.L. As a result, the conventional proton dip model is challenged for any possible parameter combination of the 3D scan. This is robust under several caveats of the fit procedure, as we have tested, see [11] for details. This result is evidence against the proton dip model completely independent of composition data.

Our result implies that the dip in the cosmic ray spectrum cannot come from pair production in a pure proton model. An obvious interpretation is that the composition of cosmic rays is heavier than protons at the highest energies, which the Auger composition measurements indicate [9].

\section{Acknowledgments}

This project has received funding from the European Research Council (ERC) under the European Union's Horizon 2020 research and innovation programme (Grant No. 646623).

\section{References}

[1] The Pierre Auger Observatory: Contributions to the 34th International Cosmic Ray Conference (ICRC 2015), 2015.

[2] Ivanov, D. et al. [Telescope Array Collaboration]. TA Spectrum Summary. PoS(ICRC2015)349, 2015.

[3] Veniamin Berezinsky, A. Z. Gazizov, and S. I. Grigorieva. Dip in UHECR spectrum as signature of proton interaction with CMB. Phys. Lett., B612:147-153, 2005.

[4] V. S. Berezinsky, A. Z. Gazizov, and S. I. Grigorieva. On astrophysical solution to ultrahighenergy cosmic rays. Phys. Rev., D74:043005, 2006.

[5] R. Aloisio, V. Berezinsky, Pasquale Blasi, A. Gazizov, S. Grigorieva, and B. Hnatyk. A dip in the UHECR spectrum and the transition from galactic to extragalactic cosmic rays. Astropart. Phys., 27:76-91, 2007.

[6] Alexander Aab et al. The Pierre Auger Cosmic Ray Observatory. Nucl. Instrum. Meth., A798:172-213, 2015. 
[7] T. Abu-Zayyad et al. The surface detector array of the Telescope Array experiment. Nucl. Instrum. Meth., A689:87-97, 2013.

[8] Belz, J. et al. [Telescope Array Collaboration]. Summary of UHECR Composition Measurements by the Telescope Array Experiment. PoS(ICRC2015)351, 2015.

[9] Porcelli, A. et al. [Pierre Auger Collaboration]. Measurements of $X_{\max }$ above $10^{1} 7 \mathrm{eV}$ with the fluorescence detector of the Pierre Auger Observatory. PoS(ICRC2015)420, 2015.

[10] R. U. Abbasi et al. Pierre Auger Observatory and Telescope Array: Joint Contributions to the 34th International Cosmic Ray Conference (ICRC 2015). 2015.

[11] Jonas Heinze, Denise Boncioli, Mauricio Bustamante, and Walter Winter. Cosmogenic Neutrinos Challenge the Cosmic Ray Proton Dip Model. Astrophys. J., 825(2):122, 2016.

[12] M. G. Aartsen et al. Constraints on Ultrahigh-Energy Cosmic-Ray Sources from a Search for Neutrinos above $10 \mathrm{PeV}$ with IceCube. Phys. Rev. Lett., 117(24):241101, 2016.

[13] Andrew M. Hopkins and John F. Beacom. On the normalisation of the cosmic star formation history. Astrophys. J., 651:142-154, 2006.

[14] Andrew M. Taylor, Markus Ahlers, and Dan Hooper. Indications of Negative Evolution for the Sources of the Highest Energy Cosmic Rays. Phys. Rev., D92(6):063011, 2015.

[15] Philipp Baerwald, Mauricio Bustamante, and Walter Winter. Are gamma-ray bursts the sources of ultra-high energy cosmic rays? Astropart. Phys., 62:66-91, 2015.

[16] Alberto Franceschini, Giulia Rodighiero, and Mattia Vaccari. The extragalactic opticalinfrared background radiations, their time evolution and the cosmic photon-photon opacity. Astron. Astrophys., 487:837, 2008.

[17] Daniel De Marco and Todor Stanev. On the shape of the UHE cosmic ray spectrum. Phys. Rev., D72:081301, 2005.

[18] M. Ahlers, L. A. Anchordoqui, M. C. Gonzalez-Garcia, F. Halzen, and S. Sarkar. GZK Neutrinos after the Fermi-LAT Diffuse Photon Flux Measurement. Astropart. Phys., 34:106$115,2010$.

[19] Kido, E. and Kalashev, O. E. et al. [Telescope Array Collaboration]. Interpretation of the energy spectrum observed with the Telescope Array surface detectors. PoS(ICRC2015)258, 2015.

[20] Kohta Murase and John F. Beacom. Neutrino Background Flux from Sources of UltrahighEnergy Cosmic-Ray Nuclei. Phys. Rev., D81:123001, 2010.

[21] Markus Ahlers and Francis Halzen. Minimal Cosmogenic Neutrinos. Phys. Rev., D86:083010, 2012.

[22] Gary J. Feldman and Robert D. Cousins. A Unified approach to the classical statistical analysis of small signals. Phys. Rev., D57:3873-3889, 1998. 